\title{
A Structure of Cross Polarization Inhibition for Narrow Wall Slot Waveguide Antenna
}

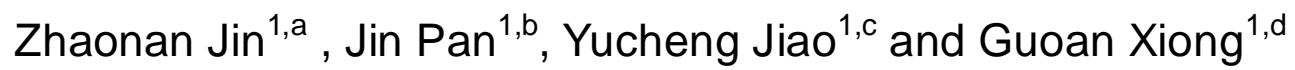 \\ ${ }^{1}$ School of Electronics Engineering, University of Electronic Science and Technology of China, \\ Chengdu 611731, China;

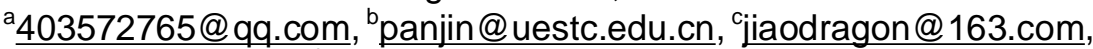 \\ gustave xiong@foxmail.com,
}

Keywords: narrow wall slot waveguide antenna, cross polarization inhibition, horn antenna

Abstract. As a kind of array antenna, narrow wall slot waveguide antenna can get desired far field pattern, by controlling the depth and inclined angle of every slot. This antenna is a linear polarized antenna with severe cross polarization which can increase sidelobe, and this cross polarization can't be eliminated by adjusting slots themselves. In many circumstances, this pattern can't satisfy application requirements for high gain, low sidelobe. Several methods of restraining cross polarization for narrow wall slot waveguide antenna are studied. A kind of easily installed and fastened structure of cross polarization inhibition with a horn antenna is designed by simulation, manufactured and tested with good effect of restraining cross polarization.

\section{Introduction}

Slotted waveguide array antenna has good properties of high gain, low sidelobe, but has a phenomenon of severe cross polarization, which will badly destroy the total gain pattern in the application. The principal polarization pattern can be controlled by designing the slots, but the cross polarization can't be eliminated through the design. Therefore, we have to add a certain structure to slotted waveguide antenna to suppress cross polarization energy. In this paper, we discuss several structures of cross polarization inhibition for slotted waveguide antenna, and design a kind of structure which is easy to be realized in engineering.

\section{Radiation Properties of Narrow Wall Slot Waveguide Antenna}

When the narrow wall of waveguide is cut off by an inclined slot, the inclined slot incises the wall current so that slot will arouse radiation field because of voltage difference between the two sides of the slot. When its narrow wall is cut off by many equally spaced slots, waveguide becomes an array antenna which is shown in Fig.1. When we design the depth and inclined angle of every slot, we can adjust amplitude distribution in order to get desired far field pattern.

Near field radiation properties of narrow wall slot waveguide antenna: the electric field radiated

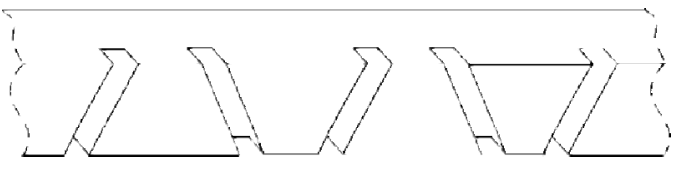

Fig. 1 narrow wall slot waveguide antenna

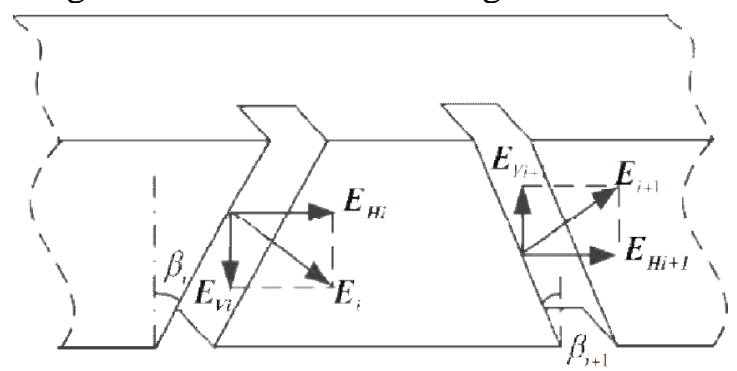

Fig. 2 method of interleaving 
Because the angle of slot is usually small $\left(\leq 15^{\circ}\right)$, the strength of horizontal component is far stronger than vertical component so that the horizontal component is principal polarization component, and the vertical one is cross polarization component. In order to weaken far field radiation of electric field vertical component, the adjacent slots are designed in the method of interleaving slotting. In this situation, electric field horizontal components are inphase, and vertical components are antiphase, as shown in Fig.2.

Far field radiation properties of narrow wall slot waveguide antenna: principal polarization pattern in the far field depends on electric field horizontal component in the near field, and it can be controlled by designing depth and inclined angle of slots; cross polarization pattern depends on electric field vertical component, and because the depth and inclined angle of every slot is different(strength of electric field for each slot is different), even if slots are designed in the method of interleaving slotting, energy in far field can't be totally offset. As shown in Fig.3, in the azimuth plane pattern, cross-polarization electric field will stack in the same phase and generate a high level at a certain angle (usually $40^{\circ} \sim 50^{\circ}$ ) off normal direction of waveguide which has a strong impact on the total gain pattern.

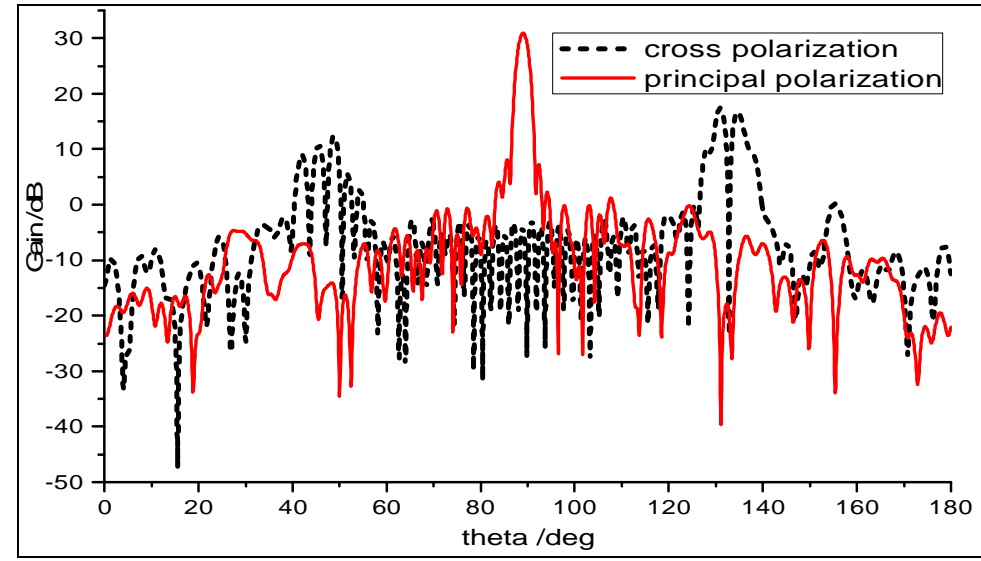

Fig. 3 far field pattern for slotted waveguide antenna

\section{Structures of Cross Polarization Inhibition and Design}

In order to reduce the influence of cross polarization in far field, we have to restrain electric field vertical component in near field. Thus, the basic strategy is to restrain vertical component in near field, but not to affect horizontal component as far as possible. There are three methods ${ }^{[1-2]}$ : adding choke groove to waveguide, adding parallel metallic grid in front of antenna array, adding parallel metallic clapboard to waveguide. This article will take the third method to design structure for cross polarization inhibition.

\section{Add choke groove to waveguide}

As shown in Fig.4, we add choke groove to the wide wall at the side of waveguide slots, and this method is not to directly affect radiation field to weaken cross polarization energy, but to change current on the surface of waveguide narrow wall ${ }^{[3]}$. When the depth of groove $\mathrm{d}$ is about $\lambda / 4$, it can be considered that surface current $J_{y}$ excited by vertical electric field is guided into a $\lambda / 4$ short circuit structure, and this part of energy is consumed to weaken cross polarization energy.

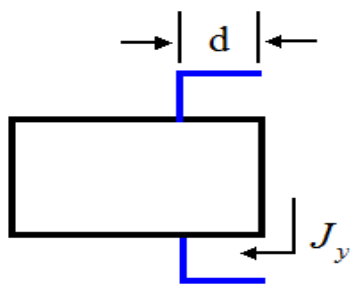

Fig. 4 side view of choke groove 
The advantages of the method are that the structure is simple and effect for cross polarization inhibition is good, but the disadvantages are that the working bandwidth is narrow, and the manufacture is relatively difficult for a large array.

Add parallel metallic grid in front of antenna array

As shown in Fig.5, we add parallel metallic grid in front of waveguide slot array, the grid strip and the narrow wall of the waveguide are vertically arranged. When the vertical component of the electric field is parallel to the metallic grid stick, the current can be excited on the metal stick, so that the electric field can be attenuated and can't pass through the metallic grid. When the horizontal component is perpendicular to the metal stick, the current is not excited, and the boundary condition of the electric field on the surface of the conductor can be satisfied, so the field can pass smoothly [4].

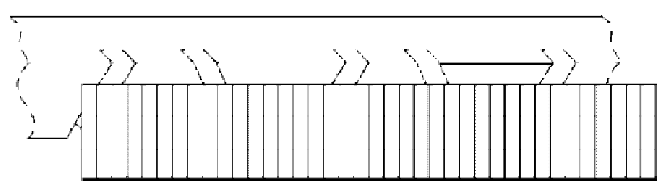

Fig. 5 waveguide slot antenna equipped with parallel metallic grid

The advantages of the method are that effect for cross polarization inhibition is best and the application range is wide; the disadvantages are that the structure is delicate, the processing is difficult, the cost is high, and the installation is not easy.

\section{Add parallel metallic clapboards to waveguide}

As shown in Fig.6, parallel metallic clapboards are equipped on both sides of every waveguide slot. For two clapboards of one slot, it can be seen as a small waveguide unit with height of a, width of $b$, depth of $h$. Let horizontally polarized waves be dominant mode for the small waveguide unit, and vertical polarized waves be a high-order mode. By adjusting the height $a$, width $b$ and depth $h$, we can filter out the vertical component, and let horizontal component pass successfully, so as to achieve effect for cross polarization inhibition ${ }^{[5]}$.

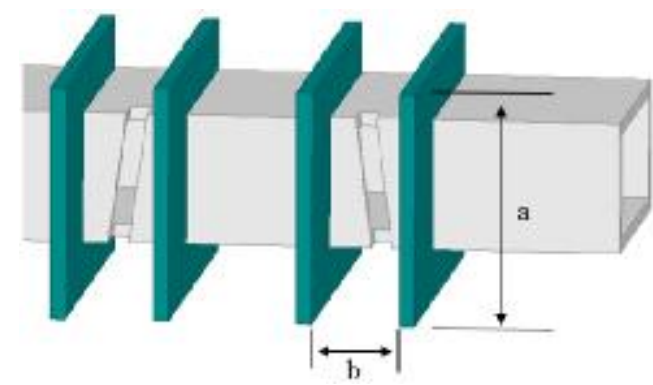

(a)

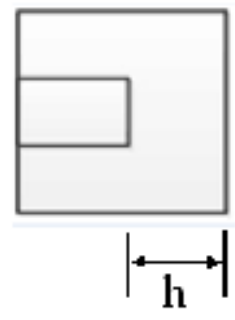

(b)

Fig. 6 waveguide slot antenna equipped with parallel metallic clapboards (a). front view (b). side view

(1) design principles for width $b$

In order not to affect the radiation of the slots, it need

$b \geq d * \tan \varphi_{\max }$.

Among them, $d$ is the size of the narrow wall, $\varphi_{\max }$ is the maximum inclined angle for all slots(approximately $15^{\circ}$ )。

In order to cut off electric field vertical component, it need

$\lambda>\lambda_{c}^{\prime}=2 * b$.

Among them, $\lambda$ is working wavelength, corresponding mode for electric field vertical 
(2) design principles for height $a$

In order to let horizontal component pass through the small waveguide unit, it need

$\lambda<\lambda_{c}=2 * a$.

Among them, $\lambda$ is working wavelength, corresponding mode for electric field horizontal component is dominant mode $T E_{10}, \lambda_{c}$ is the cut-off wavelength of $T E_{10}$ mode.

(3) design principles for depth $h$

In order to ensure that the vertical component can be sufficiently attenuated and the impedance matching can be guaranteed, it need

$h=\lambda_{g}^{\prime} / 2$.

Among them, $\lambda_{g}^{\prime}$ is waveguide wavelength of the small waveguide unit.

The advantages of the method are simple structure, easy manufacture, and good effect for cross polarization inhibition. The disadvantages are huge volume, and difficulty in installing and fixing.

In practical engineering, waveguide slot antenna is placed horizontally which can control azimuth plane pattern, but elevation plane beamwidth can't be controlled by designing waveguide slots. Common method is to add an H-plane horn antenna to the waveguide which can compress beamwidth, and increase the gain. In order to fit the inner wall of the horn, the metal clapboard is designed to be divergent shape, as shown in Fig.7. The design parameters are the thickness $k$, the distance $b$, and the depth $h$.

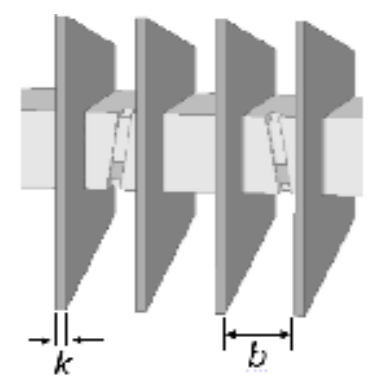

(a)

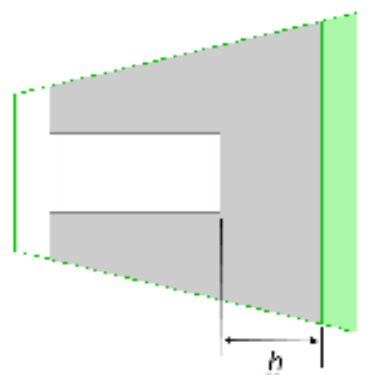

(b)

Fig. 7 waveguide slot antenna equipped with horn antenna and parallel metallic clapboards

(a). front view (b). side view

\section{Simulation Design and Process Testing}

Working frequency is $9.5 \mathrm{GHz}(\lambda=c / f=31.57 \mathrm{~mm})$, and the number of slots is 20 . The initial parameters are as follows: thickness $k=1.5 \mathrm{~mm}$, distance $b=15 \mathrm{~mm}<15.78 \mathrm{~mm}=\lambda / 2$, depth $h=\lambda / 2=15.78 \mathrm{~mm}$.

Through the optimization design of the electromagnetic full wave simulation software HFSS, the final parameters are $k=1.5 \mathrm{~mm}, \quad b=12.5 \mathrm{~mm}, h=15 \mathrm{~mm}$. Simulated far field pattern is shown in Fig.8, cross polarization energy is weaken strongly, cross polarization levels are decreased in all angles, and the inhibition reaches $45 \mathrm{~dB}$. 


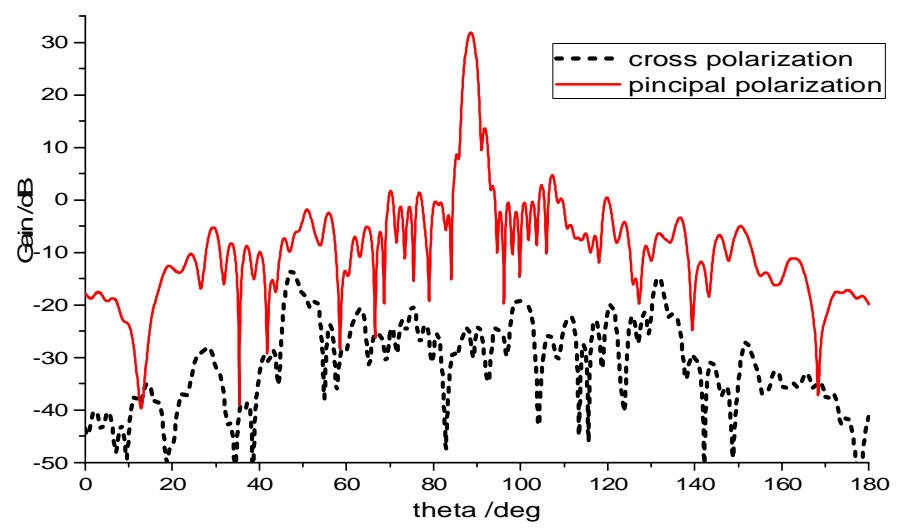

Fig. 8 far field pattern equipped with metallic clapboards

In practical engineering, the horn has a certain thickness. Thus, we can groove on the horn, add rectangular protrusions on both sides of clapboards, and install the protrusions into the horn grooves. The actual clapboard is shown in Fig.9.

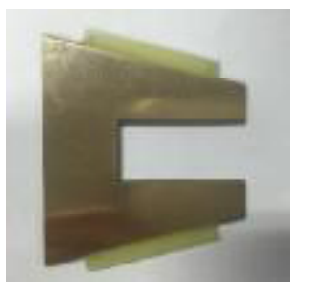

Fig. 9 actual clapboard

Fig.10 and Fig.11 are the comparison between simulation and test (normalized according to principal polarization), cross polarization inhibition reaches a relatively good effect.

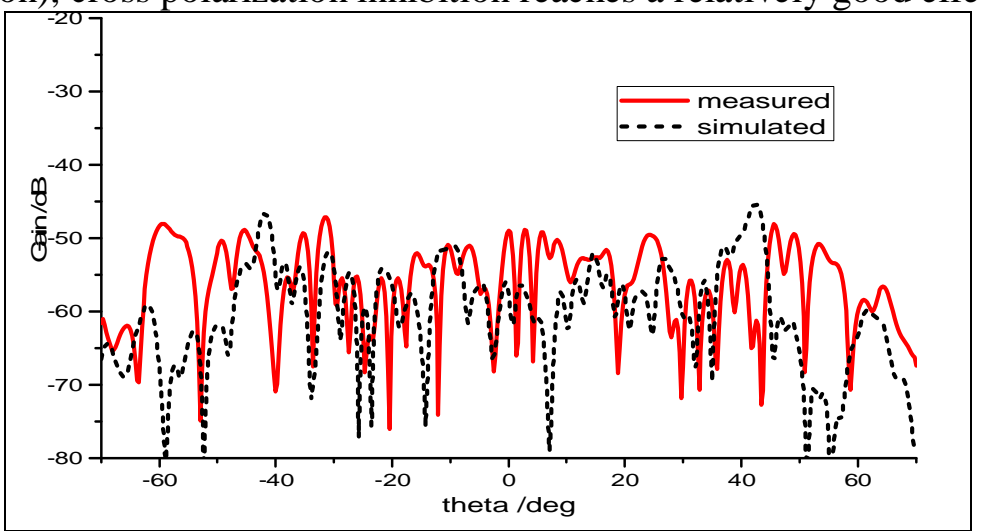

Fig. 10 far field pattern for principal polarization

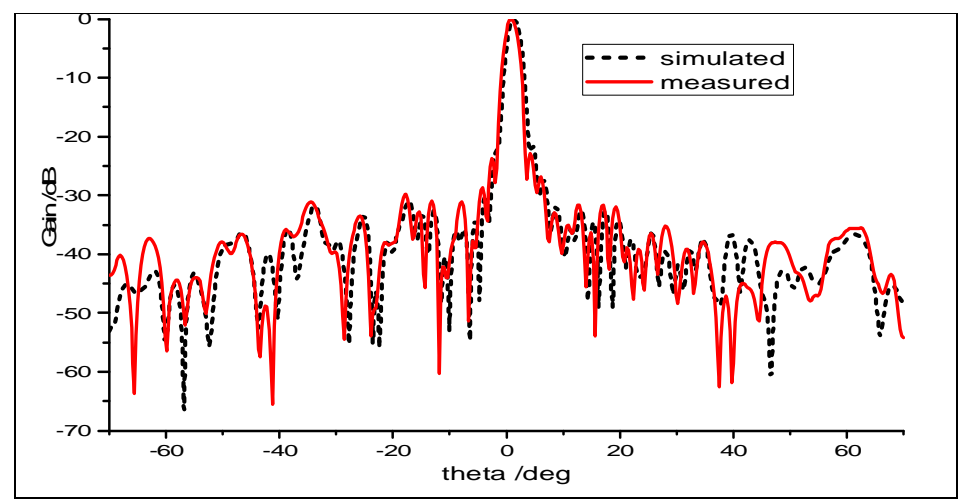

Fig. 11 far field pattern for cross polarization 


\section{Conclusions}

In this paper, three cross polarization inhibition methods for slotted waveguide array antenna is discussed, and parallel metallic clapboard is chosen as polarization filter.

Parallel metal clapboards are not easy to align and fix, which is a big problem in engineering. In this paper, we add a horn antenna to the waveguide to increase the gain, design a polarization filter which is easy to align and install with good test result.

\section{References}

[1] Xiaopeng Chen, Wenjun Chen: A Method to Suppress the Cross Polarization of Waveguide Narrow Slot Array Antenna. Radar and Countermeasure, 2014(1), P.22-34 (in Chinese)

[2] Miyuan Deng: Research on the X-Band Low Sidelobe Narrow Wall Slotted Waveguide Array Antenna. University of Electronic Science and Technology of China, Chengdu, 2013 (in Chinese)

[3] Kai Wang: Design for C-Band Narrow Wall Slot Antenna. University of Electronic Science and Technology of China, Chengdu, 2010 (in Chinese)

[4] Shuanglin Wan, Peiguo Liu: Study on the Shielding Effectiveness of Metal Mesh to Linear Polarized Electromagnetic Wave. Safety and Electromagnetic Compatibility, 2010(2), P.66-68 (in Chinese)

[5] Xianben Chen: Design of the Slot Antenna for Harbor Radar. Journal of Shanghai Ship and Shipping Research Institute, 1984 (1) (in Chinese) 